CERN - AB DEPARTMENT

CERN-AB-2006-022

\title{
RESONANT MULTI-TURN EXTRACTION: PRINCIPLE AND EXPERIMENTS
}

\author{
S. Gilardoni, M. Giovannozzi, M. Martini, E. Métral, P. Scaramuzzi, R. Steerenberg and A.-S. Müller ${ }^{1)}$
}

\begin{abstract}
Recently a novel approach to perform multi-turn extraction was proposed based on beam splitting in the transverse phase space by means of trapping inside stable islands. In addition to numerical simulations, aimed at assessing the feasibility of the proposed technique, an experimental campaign was launched since the year 2002 at the CERN Proton Synchrotron. During the year 2004 run, a high-intensity single-bunch beam was successfully split and the generated beamlets separated without any measurable losses. The underlying principle will be presented and discussed in details in this paper, as well as the latest experimental results and future applications.
\end{abstract}

Invited paper at the High Intensity Beam Dynamics Workshop COULOMB'05, Senigallia, Italy, September 12-16, 2005

Geneva, Switzerland

June 29, 2006

1) ISS, Forschungszentrum Karlsruhe, Germany 


\title{
Resonant Multi-Turn Extraction: Principle and Experiments
}

\author{
S. Gilardoni ${ }^{\text {a }}$, M. Giovannozzi ${ }^{\text {a*}}$, M. Martini ${ }^{\mathrm{a}}$, E. Métral ${ }^{\mathrm{a}}$, P. Scaramuzzi ${ }^{\mathrm{a}}$, R. Steerenberg ${ }^{\mathrm{a}}$ and
} A.-S. Müller ${ }^{b}$

${ }^{a}$ AB Department, CERN, 1211 Geneva 23, Switzerland

${ }^{\mathrm{b}}$ ISS, Forschungszentrum Karlsruhe, Germany

Recently a novel approach to perform multi-turn extraction was proposed based on beam splitting in the transverse phase space by means of trapping inside stable islands. In addition to numerical simulations, aimed at assessing the feasibility of the proposed technique, an experimental campaign was launched since the year 2002 at the CERN Proton Synchrotron. During the year 2004 run, a high-intensity single-bunch beam was successfully split and the generated beamlets separated without any measurable losses. The underlying principle will be presented and discussed in details in this paper, as well as the latest experimental results and future applications.

\section{Introduction}

Since the year 2001, intense efforts were dedicated to the study of a novel technique to perform multi-turn extraction from a circular particle accelerator. The principle of the novel technique relies on the use of nonlinear magnetic fields (sextupolar and octupolar) to generate stable islands in the horizontal transverse phase space $[1,2]$. By means of an appropriate tune variation, a specific resonance is crossed, the fourth-order in the case under study, and the beam is split by trapping inside the stable islands moving from the origin of phase space towards higher amplitudes. A good model consists in choosing a simple FODO cell with a sextupole and an octupole located at the same longitudinal position, both represented in the single-kick approximation [3]. For the application under study, only the horizontal plane is relevant. Hence, the dynamics of such a system is generated by a $2 \mathrm{D}$ one-turn transfer map, which can be computed and it turns out to be a polynomial map of the form:

$$
\left(\begin{array}{c}
\hat{X} \\
\hat{X}^{\prime}
\end{array}\right)_{n+1}=R\left(\omega_{n}\right)\left(\begin{array}{c}
\hat{X} \\
\hat{X}^{\prime}+\hat{X}^{2}+\kappa \hat{X}^{3}
\end{array}\right)_{n},
$$

\footnotetext{
${ }^{*}$ Corresponding author: massimo.giovannozzi@cern.ch
}

where the coordinates $\hat{\mathbf{X}}=\left(\hat{X}, \hat{X}^{\prime}\right)$ are adimensional normalised coordinates [3], $R(\omega)$ represents a rotation matrix of an angle $\omega=2 \pi \nu$ and $\kappa$ depends on the ratio between the strength of the sextupole and the octupole with a weight given by the value of the optical beta-function at the location of the nonlinear magnetic elements $[2,3]$, namely

$$
\kappa=\frac{2}{3} \frac{K_{3}}{K_{2}^{2}} \frac{1}{\beta} \quad \hat{\mathbf{X}}=\lambda \hat{\mathbf{x}} \quad \lambda=\frac{1}{2} K_{2} \beta^{3 / 2}
$$

where $\hat{\mathbf{x}}=\left(\hat{x}, \hat{x}^{\prime}\right)$ stand for normalised CourantSnyder coordinates. It is important to stress that the angle $\omega$ is indeed a function of the turn number. In all the plots shown in this article, as well as in the numerical and analytical computations reported here, the special adimensional normalised coordinates are used.

The evolution of the beam distribution during the resonance crossing is shown in Fig. 1. When the tune is changed, the islands move through the phase space region where the charged particles sit and a certain fraction is trapped inside the islands. At some stage a complete separation between the beamlets and the central core occurs and the distance between the beamlets can be increased at will by simply acting on the tune. It is worthwhile stressing that the beam after trapping has a peculiar structure as it is made by 
2

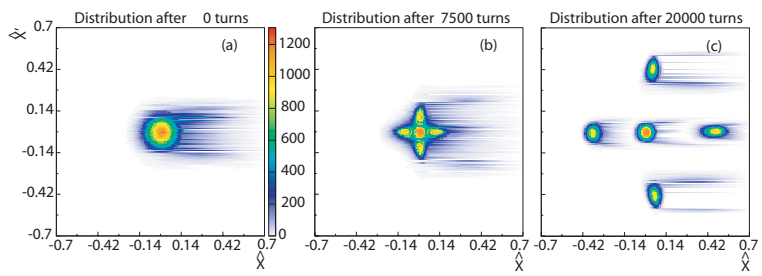

Figure 1. Evolution of the beam distribution during resonance crossing: the initial state is represented by a bi-Gaussian beam (a), at resonancecrossing some particles are trapped inside the moving islands (b), at the end of the process, the particles trapped in the islands are moved towards higher amplitudes (c).

two disconnected parts: the beamlets, which are indeed one single structure closing-up after four machine turns (see Fig. 2), and the central core. The islands' phase at two key PS sections, namely

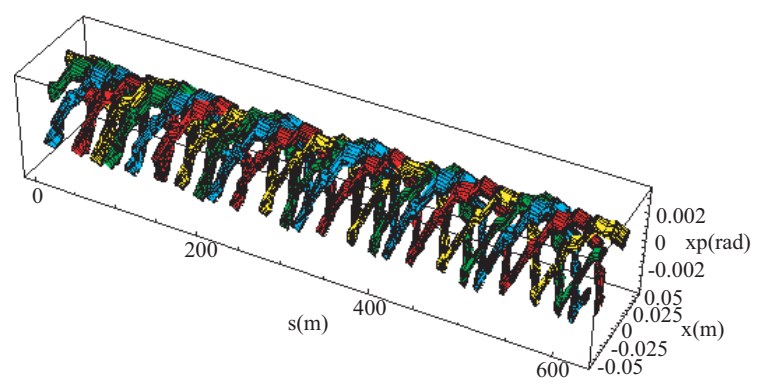

Figure 2. 3D view of the beamlets along the circumference of the CERN Proton Synchrotron (PS) machine. The fifth beamlet, i.e. the beam core, is not shown here.

the extraction septum location and the one where the wire scanner to measure the horizontal beam profile is installed, is shown in Fig. 3. The idea behind this process is that such a beam splitting in the transverse phase space can be used to perform multi-turn extraction. In fact, once the various beamlets are separated, the whole structure can be pushed towards an extraction septum by means of a closed slow bump. Then, kicker magnets generate a fast closed bump and one island jumps beyond the septum blade so that the beamlets are extracted out of the machine in four turns. The fifth beamlet, i.e. the beam core, is extracted
M. Giovannozzi et al.

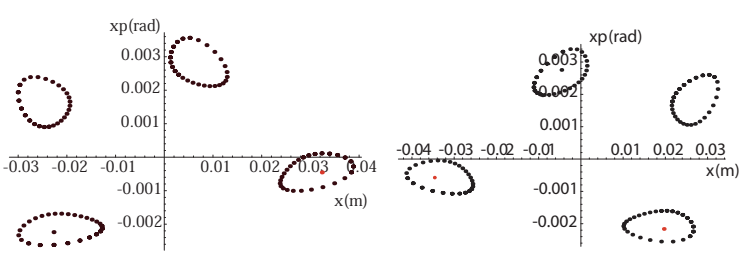

Figure 3. Shape of the stable islands at the extraction section (left) and the section where the wire scanner used for profile measurements (see next Sections) is located (right).

using a classical single-turn extraction.

The choice of the resonance to be crossed is completely arbitrary: the use of a fourth-order resonance is dictated by the CERN-specific application. The extraction mode from the PS to the Super Proton Synchrotron (SPS) foreseen to deliver the high-intensity proton beam for the planned CERN Neutrino to Gran Sasso (CNGS) experiments [4] is the so-called Continuous Transfer (CT) [5]. The beam is sliced onto an electrostatic septum and it is transferred to the SPS in five turns. The main drawback of such an extraction technique is the high losses in the septum, generating serious problems for the handson maintenance of the device. Furthermore, the extracted slices feature different shapes in phase space, thus inducing betatron mismatch at injection in the receiving machine and, eventually, emittance blow-up [6,7]. These points represent serious obstacles for the planned intensity upgrade for the CNGS beam [8]. The goal for this study is to achieve beam splitting with virtually no losses of a beam made by eight bunches, accelerated to $14 \mathrm{GeV} / c$, of about $5-6 \times 10^{12}$ protons/bunch.

\section{Further Developments}

\subsection{Analysis of the Capture Process for the Fourth-Order Resonance}

Following the first encouraging results $[9,10]$ a series of studies were launched to analyse in more details the capture and trapping process in view of improving the control of the parameters of the beamlets. This goal was tackled using two complementary approaches: a numerical and an analytical one. In the first case, the simple model 
described in Eq. (1) is used to perform numerical simulations by varying a number of key parameters such as the functional dependence of the linear tune on the number of turns, the strength of the nonlinear elements, and the parameters of the initial distribution, e.g. form of the distribution, Gaussian or uniform, and its emittance. As far as the tune-dependence on the number of turns is concerned, two models were used, i.e. a linear dependence or a power law. No major differences are found for the two options and in all the simulations reported in this paper a linear dependence is used. In Fig. 4 an example of the simulation results is reported. In the real application of this method, it is important to have a uniform sharing of initial intensity and emittance among the five beamlets after the trapping process. Ideally, one aims at having $20 \%$ of the initial intensity in each beamlet as well as $20 \%$ of the initial emittance. In the simulations discussed here, the fraction of trapped particles and the emittance of each beamlet are computed as a function of the sigma of the initial Gaussian distribution. All the other parameters are kept fixed for this study. The different markers refer to the five beamlet (the one marked Isl \# 5 is indeed the central core). It is apparent that increasing the sigma of the initial beam distribution has a positive impact on both intensity and emittance sharing. This is a consequence of the fact that the islands have a small size near the origin, hence they tend to capture fewer particles in the region with the highest density. Therefore, increasing the sigma means increasing the particle's density at larger amplitude, where the islands are bigger. An important point is that the ideal value of $20 \%$ sharing among the beamlets seems to be within reach by properly tuning the appropriate parameters. On the theoretical side intense efforts were devoted in computing the islands parameters, e.g. width, position, and secondary frequency, as a function of the linear tune and the strength of the nonlinear elements $[10,11]$. This was achieved by using the normal forms approach [3]. Analytical formulae linking the islands parameters, to $\omega, \kappa$, the detuning term $\Omega_{2}$, the resonant term $u_{0,3}$, and the distance from the resonance $\epsilon$ are reported in the following. The distance of the stable fixed

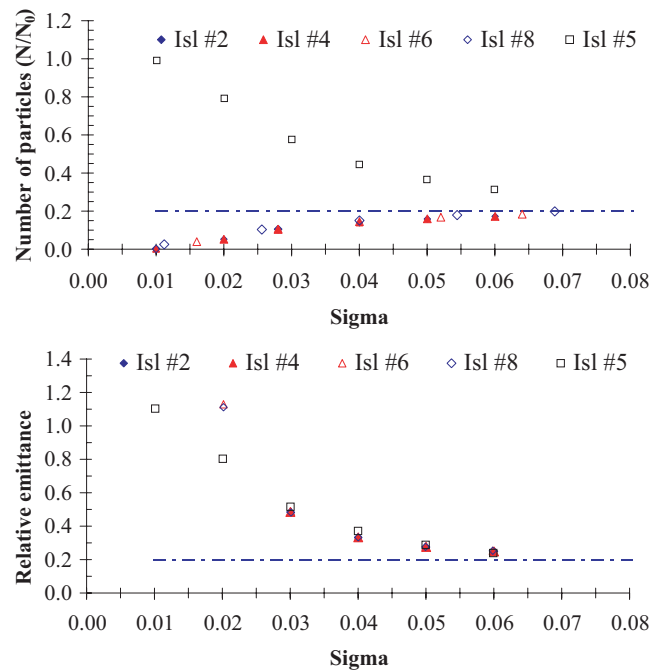

Figure 4. Results of numerical simulations concerning the intensity trapped in each island vs. the sigma of the initial bi-Gaussian distribution (upper) and the emittance of each beamlet vs. the sigma of the initial bi-Gaussian distribution (lower). The dot-dashed line represents the goal of equal sharing of both intensity and emittance.

point from the origin, $\rho_{+}$and the maximum distance between separatrices, $\Delta$, can be expressed as:

$\rho_{+}=-\frac{\epsilon}{\Omega_{2}+2 \epsilon\left|u_{0,3}\right|} \quad \Delta=4 \sqrt{\left|\frac{\epsilon}{\Omega_{2}}\right|\left|u_{0,3}\right| \rho_{+}^{2}}$

where

$$
u_{0,3}=\frac{i}{16} e^{i \omega}\left(\cot \frac{\omega}{2}-\cot \frac{3 \omega}{2}-2 \kappa\right)
$$

and

$$
\Omega_{2}=-\frac{1}{16}\left(3 \cot \frac{\omega}{2}+\cot \frac{3 \omega}{2}\right)-\frac{3}{8} \kappa .
$$

The island surface $\Sigma$ and the secondary frequency $\omega_{\text {sec }}$ are respectively

$$
\Sigma=16 \sqrt{\left|\frac{\epsilon}{\Omega_{2}}\right|\left|u_{0,3}\right| \rho_{+}^{2}} \quad \omega_{s e c}=\frac{\Sigma}{4}\left|\Omega_{2}\right| .
$$

A first application of these formulae is the control of the islands' size during the transport towards higher amplitudes. A crucial point in the 
proposed technique is that, once the beamlets are created, they should be separated to leave enough space for the blade of the extraction septum, which should not intercept any particle. This goal is achieved by changing the tune, so that the islands move towards higher amplitudes: if this is performed slowly enough, the particles trapped inside move with the islands. The key issue is that the islands' size increases with the value of the linear tune. Hence, the final value of the emittance of the beamlets increases too. The knowledge of the analytical dependence of the islands' size on the tune and on the strength of the nonlinear elements allows finding a way to move the beamlets while keeping the surface constant. In Fig. 5 an example is shown. To achieve the optimal control it is necessary to vary the strength of the sextupoles and octupoles at the end of the capture process, just before the transport towards higher amplitudes starts (left). In the centre part, the islands' surface is shown and it is clearly kept constant once the fractional part of the linear tune is higher than 0.256. Correspondingly (right) the islands centre continues moving, even though at a lower speed, when the surface is kept constant. It is planned to test these results by means of numerical simulations to evaluate the impact on the properties of the generated beamlets.
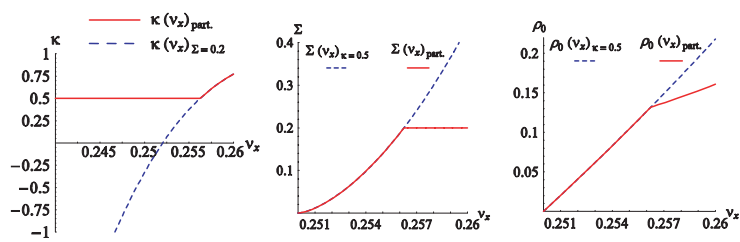

Figure 5. Example of control of the islands' size while moving them towards higher amplitudes. The solid line represents a solution with constant island surface, while the dashed line corresponds to a constant value of $\kappa$.

\subsection{Extension to Other Resonances}

Indeed, a rather straightforward extension of the proposed method to perform multi-turn extraction from a circular accelerator consists in crossing a different resonance, hence generating a different number of beamlets at the end of the splitting process [12]. The most natural alternative to the fourth-order resonance is to use the third-order one. This has also another important consequence. In fact, the fourth-order is a stable resonance, which means that particles sitting near the origin of phase space will not be trapped by the moving islands. Therefore, for a stable resonance of order $n, n+1$ beamlets will be generated. On the other hand, when the resonance is unstable, particles will be pushed away from the origin and will fill the islands. Thus, for an unstable resonance of order $n, n$ beamlets will be generated. Another interesting side effect is that the potential difference in terms of sharing particle and emittance between the central and the other beamlets is not present in this case.

Of course, the method can be generalised also to other resonances. It is worthwhile stressing that when the resonance order is increased, the islands' size tends to become smaller, thus implying that there will be fewer and fewer particles trapped within. Furthermore, there will be less room for locating the blade of an extraction septum without intercepting any particle. A detailed discussion of this application can be found in Ref. [2].

\section{Experimental Results}

\subsection{Measurement Strategy}

In parallel with the computational and theoretical analysis of the problem, an intense experimental campaign was launched since the end of year 2001 [13-16] at the CERN PS. This entailed the development of new measurement systems, such as the turn-by-turn orbit measurement system $[17,18]$, as well as the installation of sextupoles and octupoles to generate the stable islands. The magnetic elements and the beam instrumentation used in the experimental campaign are shown in Fig. 6. The tune is changed by means of two families of focusing and defocusing quadrupoles. Two sextupoles and one octupole are used to generate the stable islands; the extraction kicker is used to displace the beam and induce betatron oscillations for phase space measurements. A wire scanner [19] is used to measure the horizontal beam profile, while two pick- 
ups are used to record the betatron oscillations. The overall strategy for the experimental cam-

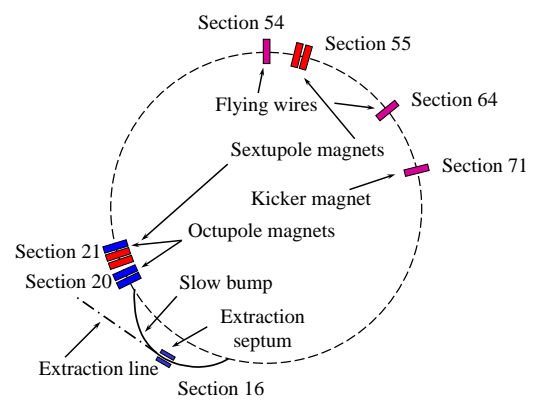

Figure 6. Schematic layout of the PS ring with the elements used for the experimental study of the novel multi-turn extraction.

paign is based on three stages.

The measurement of the phase space topology is performed by displacing a low-intensity, singlebunch, pencil beam, by means of the extraction kicker. The betatron oscillations measured by two pickups are recorded on a turn-by-turn basis and analysed to detect the presence of stable islands. Normally, the position signal features a decoherence due to beam filamentation induced by nonlinear effects and chromaticity. Whenever the beam is displaced inside one island, the natural decoherence is almost completely suppressed. An example of the measurement results is shown in Fig. 7 (see also Ref. [13] for a detailed account). From the first part of the time-series it is possible to measure the so-called secondary frequency [3] or island tune [20]. Furthermore, it is possible to scan over the amplitude inside the island to evaluate a sort of detuning curve $[10,11]$. The result of such a type of measurement is shown in Fig 7 (right). The information from the two pickups is combined to reconstruct the transverse phase space topology (Fig 7 left).

Trapping with a low-intensity beam is the key measurement. In fact, the verification of the splitting due to resonance-crossing is usually performed with a low-intensity, single-bunch beam first. This has the advantage of suppressing any possible effect due to Coulomb interaction between the protons in the bunch. A special care is devoted at the level of the PS-Booster ring, the
PS injector, when generating this special beam. In fact, as already mentioned, the larger the horizontal emittance of the initial beam, the more efficient the trapping is. Therefore, the beam is artificially blown-up in the horizontal plane, while keeping the vertical emittance as small as possible. Ideally, the horizontal emittance should have as much as possible a value similar to that of the high-intensity beam, while the vertical one should be similar to that of the pencil beam used for the phase space measurement. The first requirement allows reproducing conditions achieved during operation with an intense beam, while the second one allows reducing the nonlinear horizontal/vertical coupling, thus facilitating the settingup during the first attempts. During this stage of
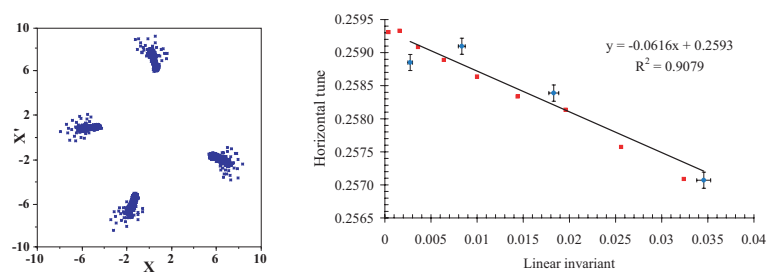

Figure 7. Results of the phase space measurements in presence of stable islands generated by means of sextupoles and octupoles. The reconstructed phase space is shown on the left and the secondary frequency vs. the amplitude inside the island is shown on the right.

the measurements the key instrument is the wire scanner [19]. It allows recording the horizontal beam profile, thus showing the details of the splitting. Examples of profile measurements can be seen in Figs. 8, 10, 11. The raw data are fitted using five Gaussian distributions whose parameters, mean, sigma, integral, reflect the properties of the beamlets.

Trapping measurement with a high-intensity beam represents the most important test. The best result achieved is shown in Fig. 8, where the intensity as a function of time is shown (left) together with the measured horizontal beam profile at the end of the capture process (right). The injected intensity is slightly above $6 \times 10^{12}$ and the intensity stays remarkably constant up to extraction, which is performed by means of a kicker in 
a single turn after having merged back the beamlets to reduce the beam size in the horizontal plane to match the mechanical aperture of the septum. In the right part of Fig. 8 the beam profile after the splitting is shown. A number of peaks are visible; in particular the central one features rather large tails. Indeed, the left tail is due to the projection of the beamlet behind (see Fig. 1, part c). Another important point is that the left-most beamlet is well-separated and the region between it and the central core is depleted. This feature is crucial for having small or no losses at all at extraction, as it guarantees no interaction between the extraction septum blade and the beam. A final test was performed to in-
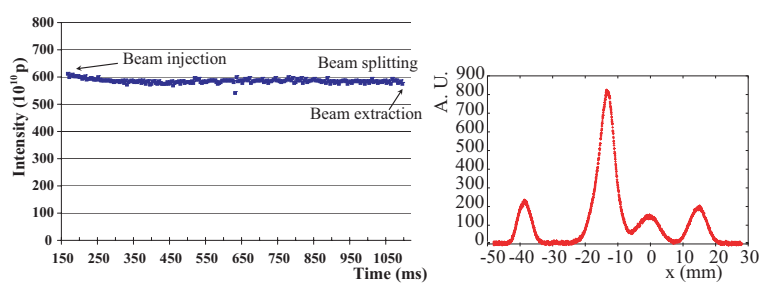

Figure 8. Best result achieved with a highintensity beam, whose intensity as a function of time (left) and horizontal beam profile at the end of the capture process (right) is shown. The profile is not centred at zero due to an instrumental offset of the wire position.

crease the fraction of particles trapped inside the islands. For this study, a special setting of the octupole was programmed: instead of keeping its strength constant all over the resonance-crossing phase, the current was suddenly increased just before resonance crossing and then gradually reduced. This generates large islands at small amplitudes, thus trapping more particles from the region where the density is high, and then keeping almost constant the islands' size. Under these new conditions it was indeed possible to increase the fraction of particles inside the islands, achieving a value of $18 \%$ against a previous value of about $13 \%$. It is worthwhile mentioning that for the optimal performance of the SPS machine, the allowed fraction of particles inside each beamlets is limited to $(20 \pm 5) \%$ : if this holds for the central core the limit for the other beamlets is instead $(20 \pm 1) \%$. The price to pay was the presence of slightly higher losses during resonance crossing up to the level of $2-3 \%$ of the total beam intensity.

As a final result the beam distribution as measured in the transfer line downstream the extraction point from the PS machine is shown in Fig. 9. An Optical Transition Radiation monitor (OTR) [21] is used to record the two-dimensional beam distribution in physical space. The peculiar

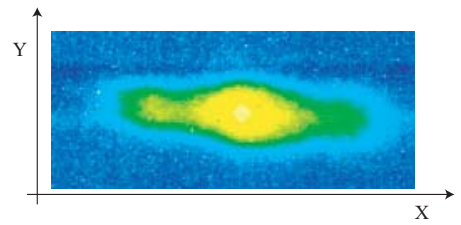

Figure 9. Two-dimensional beam distribution in physical space of the split beam in the transfer line downstream of the PS extraction point.

shape of the beam distribution is clearly visible: the two lateral peaks represent the projection in the physical space of the beamlets.

The main parameters of the single-bunch beams used in the experimental campaign are summarised in Table 1.

Table 1

Parameters of the three single-bunch beams used for the experimental tests of the novel multi-turn extraction.

\begin{tabular}{cccc}
\hline Beam type & $\begin{array}{c}\text { Intensity } \\
(\mathrm{p} / \text { bunch })\end{array}$ & $\begin{array}{c}\epsilon_{H / V}^{*}(\sigma) \\
(\mu \mathrm{m})\end{array}$ & $\begin{array}{c}\Delta p / p(\sigma) \\
10^{-3}\end{array}$ \\
\hline $\begin{array}{c}\text { Low-intensity, } \\
\text { pencil beam }\end{array}$ & $5 \times 10^{11}$ & $2.3 / 1.3$ & 0.25 \\
$\begin{array}{c}\text { Low-intensity, } \\
\text { large } \epsilon_{H}^{*}\end{array}$ & $5 \times 10^{11}$ & $6.2 / 1.6$ & 0.25 \\
High-intensity & $6 \times 10^{12}$ & $9.4 / 6.4$ & 0.60 \\
\hline
\end{tabular}

\subsection{Special Measurements}

In addition to the measurements performed to establish the feasibility of the proposed method, a number of detailed measurements were performed to study the dependence of the beamlets' parameters, such as fraction of captured particles, position, width, on the nonlinear parameters and, what is even more important, on the way the resonance in crossed.

During such a test the resonance was crossed twice with the aim of bringing back the beamlets towards the centre core. In case the resonance 
crossing is slow enough, one could argue that it should be possible to end up in a state not too different from the initial one. The crossing speed was the same in both directions and after the first resonance crossing, the tune was kept constant for a period of $20 \mathrm{~ms}$ or $180 \mathrm{~ms}$ (see Fig. 10 centre part for a sketch of the measurement principle). The horizontal beam profile was measured at three different moments: before the first resonance crossing, after the first resonance crossing when the tune was constant and the beam split, after the second resonance crossing. The crossing speed is changed and the profiles for many values of the crossing speed recorded. When a fast cross-

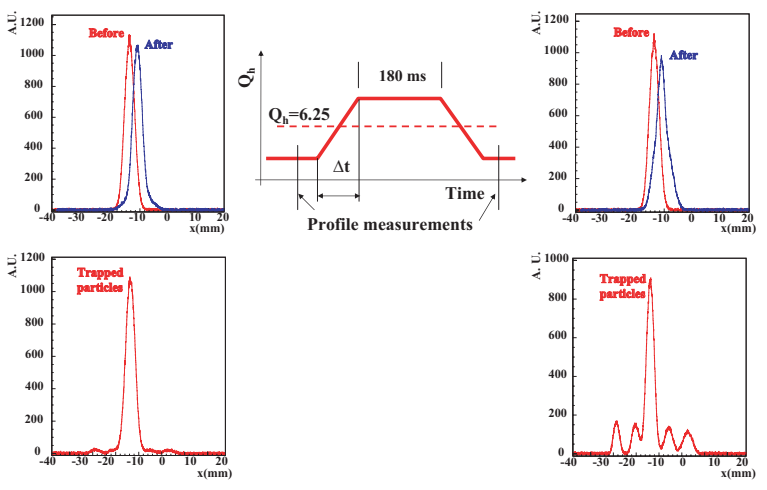

Figure 10. Results of the dependence of the beamlets parameters on the resonance-crossing speed. The principle of the measurement is shown on the centre. The horizontal profiles for a fast crossing $(5 \mathrm{~ms})$ are shown on the left, while the profiles on the right correspond to a slow crossing $(90 \mathrm{~ms})$. The profiles are not centred at zero due to an instrumental offset of the wire position.

ing occurs, almost no particles are trapped inside the moving islands and the final profile resembles very much the initial one. On the other hand, when a slow crossing occurs, many more particles are trapped inside the islands, but the final beam profile is no more Gaussian and differs from the initial one. The hypothesis is that the tails are generated by the beamlets when they are put back into the central core. As long as the crossing time is longer than $50-70 \mathrm{~ms}$ the fraction of particles trapped inside the beamlets stays constant and the final profile features non-Gaussian tails.
The situation does not change quantitatively in case the period when the tune is kept constant is reduced drastically: the process seems to be always non-reversible.

The influence of the octupole strength on the number of captured particles is the last example of detailed measurement presented in this paper. The maximum negative current is changed. The influence of the octupole is twofold: it changes the islands' size and it varies the detuning with amplitude, thus moving the islands' centres. The results are shown in Fig. 11. The increase of the number of particles trapped when the strength of the octupole is reduced is clearly visible (upper left). At the same time, the fraction of particles remaining in the central core is reduced (lower left). Two points are clearly outliers: the fit procedure failed for those two cases because of the too low intensity captured in the islands. The horizontal beam profile measured at the end of the splitting process is shown in Fig. 11 (right). The impact of the octupole on the fraction of particles trapped, as well as on the beamlets' position is clearly visible.
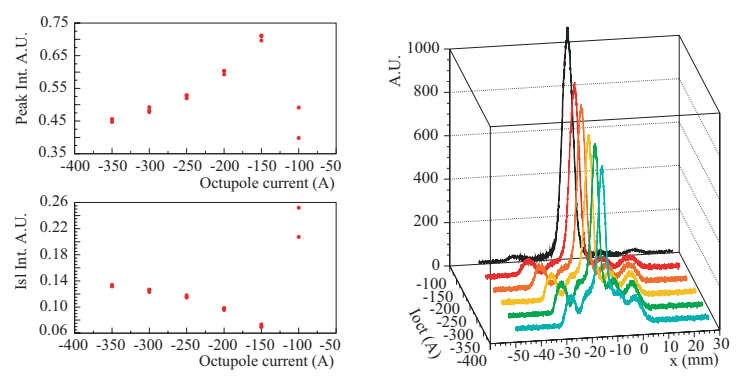

Figure 11. Results of the influence of the octupolar strength on the fraction of particles trapped in the beamlets (upper left) and in the central core (lower left). A series of horizontal beam profiles measured for different values of the octupolar strength are also shown (right part).

\section{Conclusions and Outlook}

The experimental campaign was completed by the end of the year 2004. During the long shutdown of the PS machine, which will be re-started in Spring 2006, the analysis of the required modifications to implement the proposed multi-turn extraction will take place. The main changes will 
involve a new layout of the slow bump used to approach the beam to the extraction magnetic septum and a new fast, closed-bump around the extraction septum. A crucial issue is the available aperture, as the situation is particularly critical in the extraction region.

A Study Group was set-up since 2003 [22] with the mandate to study both the theoretical, experimental, and implementation aspects of the proposed multi-turn extraction. The activity of the Study Group was prolonged in 2004 and the conclusions will be published in Fall 2005. Tests with beam will be resumed in 2006 to study how to reduce the losses observed during resonance crossing when the fraction of particles captured inside the beamlets is increased. By 2007 the new layout of the slow bump should be operational, while the fast bump should be installed and commissioned in 2008 at the latest. Therefore, the commissioning of the proposed multi-turn extraction will take place in 2008. During that year, it is foreseen to leave in operation the hardware related with the present CT extraction, to allow delivering beam to the CNGS experiments, while commissioning the novel multi-turn extraction. Finally, upon completion of this crucial stage, the CT will be decommissioned and the novel multiturn extraction will be the only means to deliver beam to the CNGS experiments.

\section{REFERENCES}

1. R. Cappi, M. Giovannozzi, Phys. Rev. Lett. 88, 104801 (2002).

2. R. Cappi, M. Giovannozzi, Phys. Rev. ST Accel. Beams 7, 024001 (2004).

3. A. Bazzani, G. Servizi, E. Todesco, G. Turchetti, CERN Yellow Report 9402 (1994), (unpublished).

4. K. Elsener (Ed.) et al., CERN 98-02 (1998) (unpublished).

5. C. Bovet et al., in Proceedings of the 1973 Particle Accelerator Conference, edited by D. W. Dupen (IEEE, New York, 1973), p. 438 , (unpublished).

6. R. Cappi, M. Giovannozzi, CERN-PS (AE) 2002-83 (2002), (unpublished).

7. R. Cappi, M. Giovannozzi, Nucl. Instrum. \&
Methods A 519, p. 442 (2004).

8. R. Cappi (Ed.) et al., CERN-PS (AE) 2001041 (2001), (unpublished).

9. R. Cappi, M. Giovannozzi, in Proc. 8th EPAC Conference, ed. by J. Poole and C. Petit-JeanGenaz (IOP, London, 2002), p. 1250, (unpublished).

10. M. Giovannozzi, P. Scaramuzzi, in Proc. 9th EPAC Conference, ed. by J. Poole and C. Petit-Jean-Genaz (IOP, London, 2004), p. 1858 , (unpublished).

11. P. Scaramuzzi, Diploma Thesis University of Milan (November 2004), (unpublished).

12. R. Cappi, M. Giovannozzi, in Proc. 2003 PAC Conference, ed. by J. Chew et al. (IEEE, New York, 2003), p. 2910, (unpublished).

13. R. Cappi et al., in Proc. 2003 PAC Conference, ed. by J. Chew et al. (IEEE, New York, 2003), p. 388, (unpublished).

14. M. Giovannozzi et al., in Proc. 9th EPAC Conference, ed. by J. Poole and C. Petit-JeanGenaz (IOP, London, 2004), p. 173, (unpublished).

15. M. Giovannozzi et al., in Proc.33 ${ }^{\text {rd }}$ ICFA Advanced Beam Dynamics Workshop on High Intensity and High Brightness Hadron Beams, ed. by I. Hofmann et al. (AIP Conference Proceedings 773, New York, 2005), p. 296 (unpublished).

16. R. Cappi et al., in Proc. 2005 PAC Conference, Knoxville, Tennessee, in press (2005), (unpublished).

17. M. E. Angoletta, A.-S. Müller, in Proc. 8th EPAC Conference, ed. by J. Poole and C. Petit-Jean-Genaz (IOP, London, 2002), p. 1948 , (unpublished).

18. M. E. Angoletta et al., in Proc. 8th EPAC Conference, ed. by J. Poole and C. PetitJean-Genaz (IOP, London, 2002), p. 1273, (unpublished).

19. C. Steinbach, M. Van Rooij, IEEE Trans. Nucl. Sci. 32, p. 1920 (1985).

20. T. Satogata et al., Phys. Rev. Lett. 68, p. 1838 (1992).

21. C. Bovet, R. Jung, LHC Project Report 3 (1996), (unpublished).

22. M. Giovannozzi (Ed.) et al, CERN-AB-2004003-ABP (2004), (unpublished). 